\title{
GEOESTRATEGIA DEL NOROESTE IBÉRICO. CASTILLA Y LEÓN EN SU RELACIÓN CON EL ESPACIO ATLÁNTICO EUROPEO
}

\author{
Fermín RODRÍGUEZ GUTIÉRREZ \\ CeCodet y Departamento de Geografía, Universidad de Oviedo \\ Rafael MENÉNDEZ FERNÁNDEZ \\ Departamento de Geografía, Universidad de Salamanca
}

Recibido: $10 / 04 / 2010$

Aceptado: 03/05/2010

RESUMEN: Las grandes líneas de fuerza de la geoestrategia europea sitúan al noroeste ibérico en un localización periférica que necesita abordar los problemas de atraso comparativo de sus regiones desde visiones y proyectos compartidos, que permitan poner en marcha estrategias de desarrollo que apuesten por su articulación en torno a la red formada por grandes nodos urbanos y ejes de relación que marcan la difusión de la actividad, la innovación y el desarrollo en el mapa de la Unión Europea. Se parte de los documentos de análisis y propuestas de desarrollo regional elaborados durante la primera década del siglo y en particular del documento del proyecto SDEA (2005).

$P A L A B R A S C L A V E$ : Estrategia de desarrollo, periferia europea, espacio atlántico, Noroeste ibérico, Castilla y León.

NORTHWEST IBERIAN PENINSULA GEOSTRATEGY AND CASTILE AND LEON

ABSTRACT: Broad lines of force in the European geostrategic locate northwest Iberian in a peripheral location which needs to address the problems concerning comparative backwardness of the regions from visions and shared projects. This projets should put in place development strategies that are committed to their coordination around the large network of urban nodes and lines of connection that make the spread of activity, innovation and development in the map of the European Union. This paper starts from the papers and regional development proposals developed during the first decade of the century and, in particular, from the project document SDEA (2005).

KEY WORDS: Development Strategy, European periphery, Atlantic Area, northwest Iberian Peninsula, Castile and Leon.

\section{GEOSTRATEGIE DU NORD-OUEST IBERIQUE ET CASTILLE-ET-LEON}

$\boldsymbol{R} \boldsymbol{E} \boldsymbol{S} \boldsymbol{U} \boldsymbol{M E}$ : Les grandes lignes de la force de l'Union européenne géostratégique situe au nord ouest ibérique dans une situation périphérique qui doit se pencher sur les problèmes de retard relatif à ses régions à partir de visions et des projets communs, ce qui mettrait en place des stratégies de développement qui se sont engagés à son coordination autour le grand réseau de noyaux urbains et les lignes de connexion qui font la propagation de l'activité, l'innovation et le développement dans la carte de l'Union européenne. On part des documents et des propositions 
de développement régional mis au point pendant la première décennie du siècle et en particulier du document du projet SDEA (2005).

MOTS CLÉ : Stratégie de développement, la périphérie européenne, l'Espace Atlantique, nordouest de la péninsule ibérique, Castille-et-Léon.

\section{GEOESTRATÉGIA DO NOROESTE IBÉRICO E CASTELA E LEÃO}

RESUMO:. As grandes linhas de força da geoestratégia europeia, classificam o noroeste ibérico como zona periférica a necessitar de resolver os problemas de atraso comparativo das suas regiões, nas suas visões e projectos partilhados, para que possam promover estratégias de desenvolvimento que apostem na rede dos grandes nós (pólos) urbanos e eixos de propagação da actividade, inovação e desenvolvimento, no mapa da União Europeia. Parte-se dos documentos de análise e propostas de desenvolvimento regional, elaborados durante a primeira década do século, em particular do documento do projecto SDEA (2005).

$\boldsymbol{P A L A V R A S C L A V E}$ : Estratégia de desenvolvimento, periferia européia, noroeste ibérico, espaço atlântico, Castela e Leão.

\section{LAS REGIONES PERIFÉRICAS EN LA ESTRATEGIA EURO- PEA DE DESARROLLO}

Castilla y León no forma parte de las regiones marítimas atlánticas pero su localización está marcada por una posición intermedia entre el área más dinámica de la península Ibérica, la aglomeración urbana de Madrid y las regiones periféricas marítimas de la fachada atlántica. Su relación geoestratégica con el ámbito centroeuropeo establece una ruptura interna, pues presenta un espacio más dinámico, el vinculado a los nodos urbanos de los grandes ejes de comunicación de Madrid con Europa, a través de Burgos y Miranda de Ebro y su conexión con Valladolid, y el eje Europa-Portugal; y por otra parte, las provincias del oeste de la Comunidad se relacionan con un territorio-problema, el vinculado a la frontera entre España y Portugal, de menor dinamismo y grado de desarrollo.

La estrategia de desarrollo de las regiones periféricas europeas ha sido abordada por numerosos estudios y análisis desde un enfoque global europeo. Especialmente interesante para conocer la España atlántica y el papel que juega en ella Castilla y León resulta el Schéma de Développement de l'Espace Atlantique-SDEA (Varios Autores, 2005), realizado en continuidad de las orientaciones del Schéma de Développement de l'Espace Conmunitaire-SDEC, en este caso para un territorio marítimo y periférico. SDEA perseguía el objetivo de ayudar a construir un modelo de desarrollo policéntrico y equilibrado del espacio atlántico europeo, actualmente caracterizado por su posición periférica y por las diferencias de grado de desarrollo respecto al espacio central. Así pues, la elaboración de SDEA supuso una continuidad de las orientaciones de SDEC, concernientes a la construcción de un modelo de 
desarrollo policéntrico y equilibrado del territorio europeo, armonizándolo con los objetivos del programa Interreg III B Atlántico -estructuración policéntrica del espacio y desarrollo de polos de competencia-.

Desde ese punto de vista, una estrategia de desarrollo para las regiones atlánticas, incluído el espacio interior peninsular de Castilla y León, debía aportar una visión territorializada del policentrismo, identificando sus condiciones de puesta en práctica al tiempo que sugería las orientaciones clave y las medidas compartidas por las administraciones competentes, en sus diferentes escalas de responsabilidad.

Los objetivos estratégicos de desarrollo se definieron en el análisis SDEA en los siguientes aspectos:

La creación de una zona de integración económica de importancia mundial

$\checkmark$ La estructuración del armazón urbano en red, vertebrando las grandes ciudades, las ciudades medias y las pequeñas urbes que organizan los territorios de dominante rural

$\checkmark$ El refuerzo de la competitividad y de la innovación en el territorio atlántico

La movilización de los actores y el refuerzo de las redes profesionales y de cooperación

Los trabajos de SDEA se realizaron en paralelo al estudio del programa europeo ESPON (Observatorio en red sobre la ordenación del territorio europeo), sobre el desarrollo policéntrico de la Unión Europea. Y ambos mantienen una línea de continuidad respecto al estudio de prospectiva "Europa 2000", realizado por la Comisión Europea para delimitar los grandes espacios de cooperación transnacional, así como del estudio de la Conferencia de Regiones Periféricas y Marítimas sobre la construcción del policentrismo en Europa.

Estos estudios apuntan a que la globalización de la economía provoca una recomposición del territorio que favorece espontáneamente a las regiones más avanzadas, las cuales disponen de las mejores ventajas comparativas. Son las que se localizan en el denominado "pentágono europeo", delimitado por las ciudades de Londres, París, Milán, Munich y Hamburgo. Lo que conlleva el riesgo de que el modelo centro-periferia se consolide en Europa, amenazando las perspectivas de integración territorial europea y generando diferentes niveles de desarticulación espacial, con territorios y sistemas urbanos cercanos al 
pentágono o a las áreas metropolitanas de las capitales nacionales que se pueden beneficiar de los efectos de difusión de las regiones centrales, mientras que las más periféricas, como las regiones atlánticas, tenderían a exponerse a un riesgo creciente de marginalización. Estas amenazas requieren de la puesta en marcha de estrategias de desarrollo policéntrico por parte de las regiones periféricas.

El trabajo de análisis y propuestas de SDEA se realizó entre enero de 2004 y junio de 2005, en tres fases: definición de la metodología, evaluación estratégica del territorio atlántico a partir de un diagnóstico de los sistemas territoriales y sus tendencias de evolución y, por último, la propuesta de un esquema de desarrollo policéntrico en el Espacio Atlántico. Con la participación activa de la mayor parte de las regiones concernidas y de sus actores socioeconómicos, y a través de varias universidades y centros de investigación, con la cooperación de la Comisión Arco Atlántico de la CRPM, la Conferencia de Ciudades del Arco Atlántico (CVAA), la Asociación de Cámaras Agrarias del Arco Atlántico (AC3A) y la Red Transnacional Atlántica (RTA) y sus estudios sobre la intermovilidad y los transportes marítimos, la seguridad marítima y la innovación. Los resultados de la cooperación existente fueron tenidos en cuenta a través de una análisis cuantitativo y cualitativo de los proyectos Interreg III B.

El territorio atlántico europeo se define en los estudios y análisis como un espacio heterogéneo, tanto en los aspectos económicos como en los geográficos y socioculturales. Su organización territorial responde a las lógicas territoriales de diferentes subconjuntos, que responden a formas de articulación diferentes, aunque tienen como característica común la ausencia de metrópolis europeas de primer rango. Presenta, en cambio, un conjunto apreciable de ciudades medias, una diversidad y riqueza de recursos naturales, culturales y saber hacer que propician que este espacio disponga de un apreciable potencial de desarrollo. La visión territorializada del desarrollo policéntrico giró en torno a cuatro ejes de trabajo fundamentales:

Los territorios y los sistemas urbanos y sus interrelaciones y dinámicas en el curso de los diez últimos años, englobando una serie de campos como la competitividad, la internacionalización, el capital humano, la conectividad, la cooperación interregional

Los sistemas productivos de los diferentes territorios atlánticos, haciendo hincapié en la economía marítima, como aspecto fundamental para el espacio atlántico

Los transportes, como dimensión fundamental para la construcción del policentrismo y para el desarrollo sostenible 
Los sistemas de gobernanza propios de cada uno de los territorios y regiones

El informe presenta la evaluación estratégica de los sistemas territoriales y urbanos atlánticos, incluyendo a Castilla y León, y expone una propuesta de organización del policentrismo atlántico europeo, como conclusión de los análisis contenidos en el diagnóstico. También incluye las recomendaciones para cada una de las regiones y espacios que componen el Espacio Atlántico, con el objetivo de reforzar los territorios y favorecer su puesta en red. Se formulan politicas territoriales y sectoriales y se sugieren posibilidades de cooperación en el seno del Espacio Atlántico.

\section{UNA ESTRATEGIA DE DESARROLLO PARA LA IBERIA ATLÁNTICA. EL PAPEL DE CASTILLA Y LEÓN}

A pesar de su carácter interior, Castilla y León tiene una evidente relación geoestratégica con las regiones ibéricas atlánticas, a partir de su localización intermedia entre la gran aglomeración de Madrid, la principal de la península y una de las grandes metrópolis de la Unión Europea, y las regiones atlánticas de España (País Vasco, Cantabria, Asturias, Galicia) y Portugal.

Esta posición intermedia ha tenido y tiene importantes efectos territoriales en una Comunidad muy extensa, que juega un papel fundamental en el mallazo de los distintos espacios regionales ibéricos. Desde el punto de vista atlántico y a partir de los análisis realizados en SDEA, Castilla y León se nos presenta como un territorio extenso, relativamente poco poblado, con áreas rurales de muy débil densidad y un pequeño conjunto de ciudades medias que ordenan el territorio, como primer nivel jerárquico, complementado por una también poco numerosa red de pequeñas ciudades, de importancia estratégica y logística para los grandes ejes de transporte, y una más numerosa constelación de pequeños centros comarcales, de población inferior a los 15.000 habitantes y funcionalidad en rápida transformación, vitales para mantener el poblamiento rural, muy afectado por la gran emigración de la segunda mitad del siglo XX que envejeció y vació extensas áreas.

Sobre el espacio de la meseta norte, Valladolid adquiere en las tres últimas décadas un papel destacado sobre el resto de ciudades medias, apoyada en el crecimiento industrial de las décadas de 1960 y 1970, reforzado más tarde por la concesión de la centralidad administrativa autonómica y la modernización económica. Su influencia y centralidad regional tiende a consolidar un entorno de mayor dinamismo regional sobre el eje Tordesillas-Valladolid-Palencia, que adquiere un cierto papel regional dominante sobre un tejido urbano en proceso de consolidación y crecimiento, marcado por la presencia e influencia de algu- 
nos corredores de transporte de gran flujo, como el Irún-Oporto (Miranda de Ebro-Burgos-Palencia-Valladolid-Salamanca). El área metropolitana de Valladolid se acerca a los 400.000 habitantes y la de influencia directa de Palencia a los 100.000 .

Sin embargo, a pesar de las tendencias centralizadoras, la meseta norte mantiene en buena medida un sistema urbano de carácter policéntrico, sin una jerarquización clara sobre un nodo preponderante, a pesar del crecimiento y centralidad de Valladolid.

Figura 1. Dinámica de la red de nodos urbanos del noroeste Ibérico (CeCodet, SDEA 2005)

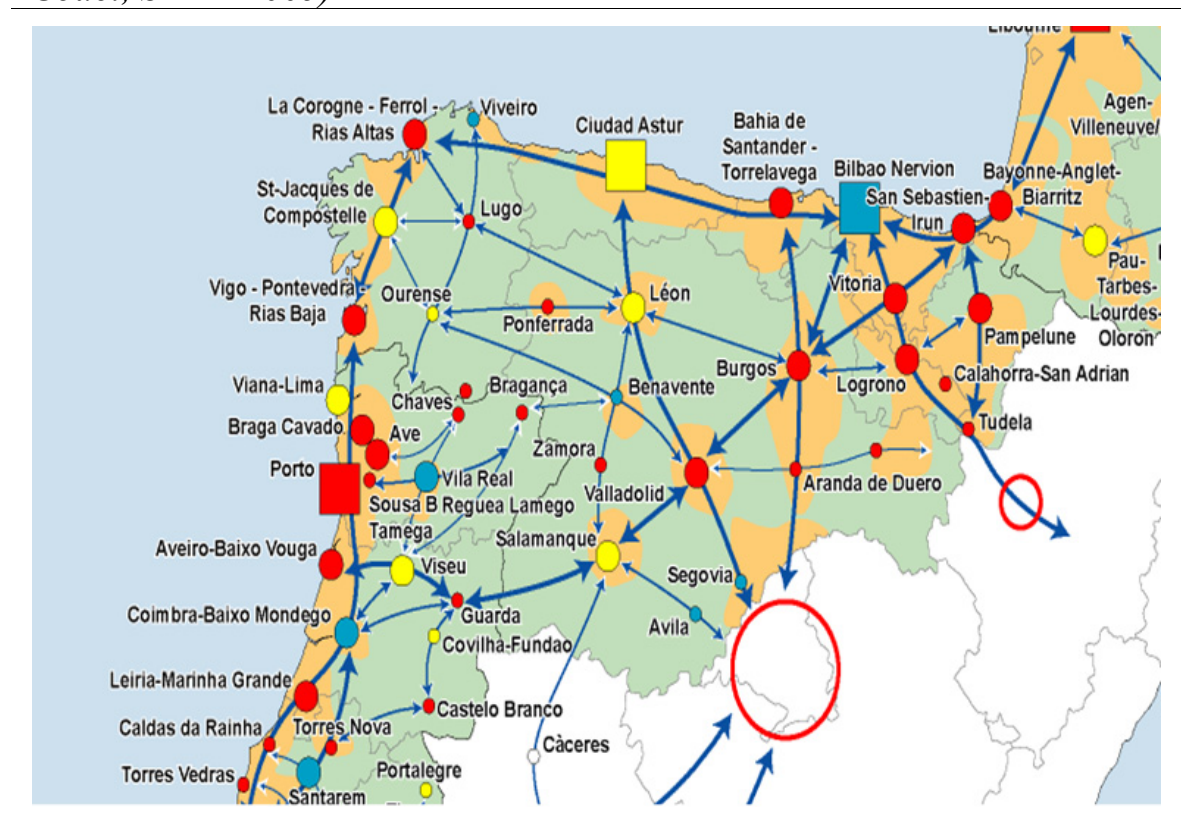

FUENTE: CeCodet, 2005. Dinámicas demográficas y económicas del NO de España $y$ Portugal

Un primer nivel jerárquico urbano está formado por la citada Valladolid, que ha tendido a relacionar con su área metropolitana a ciudades y centros comarcales más o menos próximos como Palencia, Medina del Campo, Venta de Baños o Tordesillas, además de los numerosos municipios metropolitanos afectados por su expansión urbana. Pero también por otra serie de ciudades que ocupan y ordenan el conjunto del territorio regional y se sitúan en torno a los 150.000200.000 habitantes: León, Salamanca y Burgos, con crecimiento como capitales provinciales, actividad industrial y terciaria y creciente difusión metropolitana sobre los municipios vecinos. Son precisamente los municipios metropolitanos 
de estas ciudades los de mayor aumento demográfico de la región en las tres últimas décadas (Laguna de Duero, Santa Marta de Tormes, San Andrés de Rabanedo,...)

A este primer nivel le seguiría otro escalón, formado por capitales de provincia con características de medias-pequeñas ciudades (Ávila, Segovia, Zamora, Soria) y por centros urbanos sin capitalidad, de área de influencia subprovincial: Ponferrada, Medina del Campo, Miranda de Ebro, Aranda de Duero, Benavente, con dimensiones entre los 20.000 y los 65.000 residentes. Tanto en un nivel como en otro, la mayoría de estas ciudades ha desarrollo, en las últimas décadas, fenómenos de difusión metropolitana sobre los municipios vecinos, mayores, lógicamente, en Valladolid y las ciudades medias pero también importantes en las ciudades medias-pequeñas.

Destaca el papel fundamental que han jugado y están jugando los grandes corredores europeos de transporte en el crecimiento y dinamismo de los principales nodos urbanos. El eje más dinámico es el que comunica Francia y Portugal y que relaciona la densa red urbana vasca y el eje París-Madrid con Portugal, a través de Miranda de Ebro (muy próxima a Vitoria), Burgos, Palencia, Valladolid, Tordesillas, Salamanca y Ciudad Rodrigo hacia Aveiro, en el eje OportoLisboa. Este eje vincula a la mayor parte de la red urbana de Castilla y León. Por otra parte Ávila, Segovia y Aranda de Duero entran a formar parte, de forma progresiva, del ámbito de influencia de la gran metrópoli madrileña.

Los centros comarcales se sitúan entre los 16.000 y los 2.000 habitantes, según sea la dimensión de sus áreas de influencia o la localización de actividades de cierta entidad. En las áreas periféricas de montaña incluso menos. Los principales son: Astorga, La Bañeza, Bembibre, Cistierna, Fabero, La Pola de Gordón, La Robla, Villafranca del Bierzo (León), Íscar, Olmedo, Peñafiel, Tordesillas (Valladolid), Belorado, Briviesca, Lerma, Medina de Pomar-Villarcayo (Burgos), Aguilar de Campoo, Carrión de los Condes, Cervera de Pisuerga, Guardo, Herrera de Pisuerga, Saldaña (Palencia), Alba de Tormes, Béjar, Ciudad Rodrigo, Guijuelo, Peñaranda de Bracamonte, Vitigudino (Salamanca), Arenas de San Pedro, Arévalo, El Barco de Ávila, Candeleda, Cebreros, Las Navas del Marqués, El Tiemblo (Ávila), Cantalejo, Cuéllar, El Espinar (Segovia), Ágreda, Almazán, Burgo de Osma, San Esteban de Gormaz (Soria), Toro y Puebla de Sanabria (Zamora). Por su parte, el poblamiento rural se reparte entre varias decenas de miles de núcleos de pequeño tamaño, en general de menos de 2.000 habitantes, si bien la mayoría no alcanzan los mil residentes. Sus 2.248 municipios representan un significativo porcentaje entre los 8.108 españoles, magnificándose el rasgo si tenemos en cuenta que más de la mitad tienen menos de 500 habitantes y la casi totalidad (94\%) no superan los 2.000 habitantes. 
Figura 2. Tipologías y tendencias urbanas de las áreas urbanas atlánticas (SDEA, 2005)

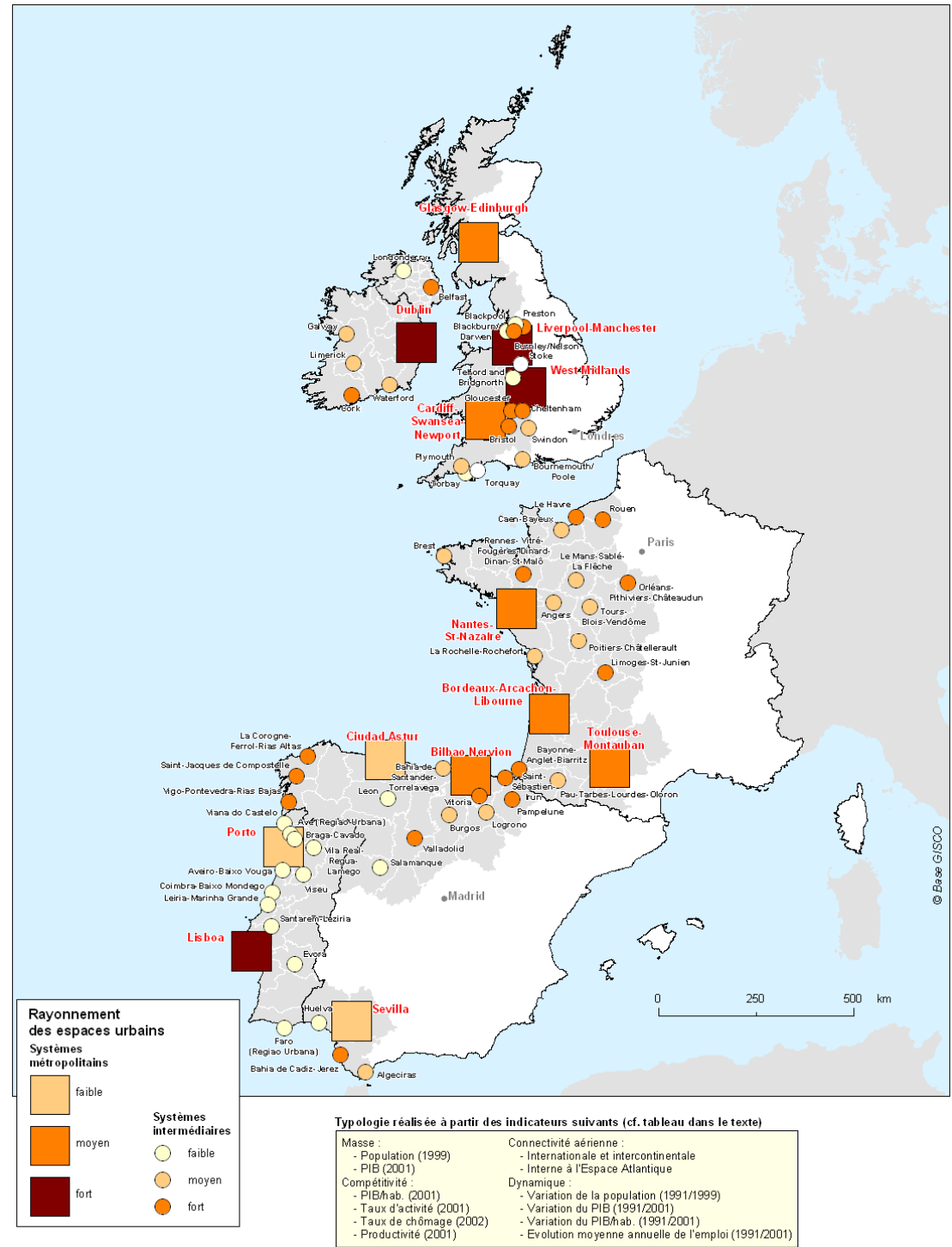

Destaca también, como ya habíamos adelantado, la ruptura desde el punto de vista del desarrollo territorial entre la parte oriental de la comunidad, en torno al eje principal de comunicación y transporte y los nodos urbanos, que se sitúa por encima de los indicadores medios de desarrollo, o próximos a ellos, de la Unión 
Europea (Burgos, Valladolid, Palencia, Soria, Segovia) y la parte occidental en el límite con Portugal, Galicia y Asturias que presenta indicadores por debajo de la media y preocupante situación.

Desde una perspectiva ibérica y europea, se forma un espacio de ruptura de orientación norte-sur que se extiende desde el límite interior de las montañas de Asturias y Galicia, por el oeste de Zamora y Salamanca y sus límites con Galicia y Portugal, y se continúa por Extremadura y la montaña de Huelva, en paralelo con el otro lado de la frontera, que ocupa buena parte del interior de Portugal. Conforma uno de los espacios más atrasados, de más debilidad demográfica y mayor estancamiento de todo el conjunto de la U.E., por lo que conforma un particular "finisterre" interior dentro del mundo atlántico europeo. Una respuesta estratégica a esta situación requeriría de una visión europea puesto que engloba a dos países y numerosas regiones. En cualquier caso, el oeste de Castilla y León, formado por las provincias de León, Zamora y Salamanca (a la que se podría añadir Ávila, al menos en parte) enfrenta de manera conjunta con Galicia y Asturias fenómenos tales como los más negativos indicadores demográficos, respecto a natalidad, mortalidad, envejecimiento, dependencia, población activa y otros. Por ello no debería olvidarse la necesidad de afrontar problemas comunes que tiene un mismo origen, de manera coordinada.

En las recomendaciones territoriales relativas al reforzamiento de los diferentes niveles de la red urbana atlántica, cobra especial importancia el apoyo a las metrópolis emergentes. La consolidación y refuerzo de los principales nodos urbanos atlánticos españoles, en el segundo escalón urbano del sistema urbano y como elementos básicos de la ordenación del territorio y de la creación y difusión de innovación y desarrollo, necesita de su adecuada conexión con la malla urbana peninsular y europea, así como del aprovechamiento de la descentralización administrativa para el incremento de sus conexiones internacionales directas. También del incremento de la relación entre los nodos urbanos atlánticos y de sus territorios rurales de influencia, a través de la incorporación del concepto de "región urbana" y de la facilitación de las relaciones e intercambios entre las ya individualizadas, como territorios de poblamiento denso, intensa actividad económica y gran volumen de flujos de interrelación.

La red urbana de Castilla y León muestra diferentes escalas jerárquicas, perspectivas y necesidades en función de sus caracteres y tendencias dominantes. Su relación con las áreas metropolitanas cantábricas tiene una especial importancia. Las áreas metropolitanas de Bilbao y Asturias constituyen aglomeraciones que han desarrollado intensos procesos de reestructuración productiva, los cuales se han traducido en unestancamiento y crecimiento relativo menor a lo largo de la década de 1980 y 1990, desde una situación anterior dominada por la industria siderometalúrgica, de fuerte crecimiento en las dos décadas anterio- 
res a 1980 y de reestructuración en las siguientes. La finalización de ese periodo y su acompasamiento a los ritmos generales de crecimiento de España se ha producido en la segunda mitad de la última década del siglo, abriéndose paso una nueva situación de crecimiento sostenido y mejora de los indicadores demográficos, de productividad, competitividad y dinamismo, ritmo mantenido hasta la actualidad y más intenso en el País Vasco y Cantabria, dada su localización favorable respecto a los grandes ejes transnacionales. La recuperación del polo vasco tiende a consolidar un tejido urbano cada vez más denso, que sobrepasa los límites entre comunidades, vinculando fuertemente la actividad de la red urbana vasca, Cantabria, Navarra, La Rioja y los nodos urbanos más próximos de Castilla y León: Miranda de Ebro y Burgos, cuya posición se revela cada vez más estratégica, sobre el eje que comunica esta extensa región urbana con Madrid y que, a su vez, forma parte del gran canal de comunicación de la aglomeración madrileña y del conjunto de España interior con Europa.

En definitiva, los nodos principales de la red urbana atlántica española deben apostar por la dinamización de los ejes peninsulares como vías de crecimiento y difusión del desarrollo y la innovación, a partir del incremento de la relación directa entre los polos principales y secundarios y la articulación interna de las áreas metropolitanas y regiones urbanas. Y ello a partir de la consolidación de los ejes que presentan un mayor dinamismo en el momento actual: IrúnValladolid-Portugal, Galicia-Oporto-Lisboa, eje cantábrico y el que relaciona Francia con País Vasco, Burgos y Madrid. Especial importancia cobra la agilización de las importantes mejoras en curso (aeropuertos, puertos, carreteras, ferrocarriles de alta velocidad) de la accesibilidad de los territorios con peor grado de conectividad y más bajos índices de desarrollo (interior de Galicia, oeste de Asturias, oeste de Castilla y León, áreas de montaña)

La utilización, en provecho propio, por parte del sistema urbano atlántico de la posición de Madrid, se revela como estrategia fundamental. Madrid ejerce una influencia creciente sobre la red urbana de Castilla y León, con efectos de difusión de gran interés si se consigue un aprovechamiento integral de los mismos, en aumento rápido en las dos últimas décadas, lo que tiene consecuencias ya visibles sobre algunas áreas urbanas de tamaño medio (Segovia, Ávila, Aranda de Duero). Pero también Madrid ejerce una amplia influencia sobre las áreas metropolitanas regionales, a través de una densa red de enlaces, caso de Valladolid, pero también de León o Burgos, que cuentan con importantes infraestructuras de transporte en construcción, como son la prolongación del ferrocarril de alta velocidad desde Valladolid a Asturias y hacia Burgos-MirandaVitoria, o las autovías Valladolid-León, Valladolid-Soria y las recién finalizadas Valladolid-Segovia y Palencia-Torrelavega. 
Las claves estratégicas de futuro para las ciudades intermedias se apoyan en el mayor papel que juegan, en la estructura administrativa española, en aquellas comunidades autónomas de pequeño tamaño, donde adquieren la condición de capitales regionales, localizan centros de poder y decisión y disponen de recursos financieros crecientes. En el caso de Castilla y León, la extensión territorial y la existencia de varios sistemas urbanos intermedios, junto al papel de las nuevas infraestructuras de transporte, privilegian el papel central de Valladolid y su relación con las ciudades medias y pequeñas próximas, en localizaciones estratégicas, especialmente aptas para la atracción de actividades logísticas (Palencia, Medina del Campo, Tordesillas).

La existencia de grandes ejes de transporte transregionales y transnacionales ha propiciado el crecimiento de las áreas empresariales localizadas en los nodos estratégicos. La difusión del crecimiento a lo largo del eje Vitoria-BurgosValladolid-Salamanca, en las tres últimas décadas, a partir del ingreso de Portugal y España en la Comunidad Europea, es un recurso de primer orden para facilitar su difusión a otros ejes de tendencias más débiles, caso de ValladolidLeón-Asturias, de la denominada Ruta de la Plata (León-Astorga-Salamanca) o de las conexiones con Galicia. Polos estratégicos y aumento de la calidad y función residencial se revelan como puntos fundamentales de trabajo en las ciudades intermedias.

La continuidad de los efectos favorables para el policentrismo de la descentralización administrativa debe impulsarse en las Comunidades Autónomas de gran extensión con el fortalecimiento del segundo escalón urbano, compuesto, fundamentalmente, por capitales de provincia, descentralizando funciones y servicios. Y ello porque el reforzamiento de las funciones de los centros administrativos de distinto nivel y su consolidación posibilitan el crecimiento de los distintos niveles jerárquicos urbanos, tendiendo a la formación de una malla equilibrada, que evita las tendencias excesivas hacia la centralización en las grandes aglomeraciones y suaviza el fuerte liderazgo que sobre el conjunto ejerce Madrid. Desde este punto de vista, es significativo que la descentralización de funciones del Estado hacia las Comunidades Autónomas no solo no ha significado merma alguna de la capacidad de crecimiento de Madrid, sino que, por el contrario ha conocido su periodo de mayor crecimiento urbano, hasta constituir uno de los polos de actividad más importantes de la Unión Europea.

La creación de nuevas capitales regionales ha tenido un efecto favorable en la dinamización de ciudades medias (Santiago de Compostela, Mérida) o en la consolidación de las que tenían la consideración de capitales provinciales (Vitoria, Santander, Oviedo, Pamplona, Logroño, Valladolid). Del mismo modo, la descentralización de funciones de las regiones hacia otros niveles administrativos debe servir para reforzar en el futuro los distintos niveles jerárquicos de la 
red urbana; en particular en aquellas áreas que más han sufrido la emigración y el declive demográfico.

Las medidas estratégicas para las ciudades medianas y pequeñas deben partir del hecho de que la red de estas urbes juega un papel fundamental en la ordenación del territorio y en la propia supervivencia de importantes espacios de baja densidad de población, especialmente en el interior (Castilla y León, Galicia interior) y en las áreas de montaña: cordillera Cantábrica, Montes de León, sistema Ibérico, sistema Central. El papel de estas ciudades en la atracción de actividad a partir de su relación con las grandes ciudades y en particular con Madrid, juega en favor de ciudades como Segovia, Ávila o Aranda de Duero.

En el caso de regiones de población más densa, la interrelación permite el crecimiento de núcleos menores, caso de las ciudades industriales vascas, de los nodos de tamaño medio del valle del Ebro (Miranda de Ebro, Calahorra, Tudela) o de ámbitos intermedios entre áreas metropolitanas, de influencia provincial o subprovincial, en el caso de Castilla y León (Zamora-Benavente, Ponferrada, Soria). También de Galicia (Ourense, Lugo y el escalón de pequeñas ciudades del interior: Verín, Monforte, Lalín, Viveiro).

Su posición central sobre territorios rurales en declive y transformación debe apoyarse a través de políticas de desarrollo rural que potencien nuevas actividades en sus áreas de influencia y del refuerzo de las actividades secundarias y terciarias vinculadas a nuevos productos y servicios y al incremento del uso de tecnologías de la información en las pequeñas y medianas empresas, así como su expansión a nuevos mercados, en algunos casos a partir de la producción de calidad vinculada a marcas territoriales.

La continuidad del proceso de descentralización administrativa debe dirigirse hacia una mejora de la dotación de recursos de esta red que permita la elaboración y puesta en marcha de planes estratégicos de desarrollo, de medidas de mejora de la accesibilidad y de incorporación de nuevas actividades de servicios avanzados que les faciliten su potenciación como núcleos urbanos, a pesar de su posición actual de dependencia respecto a los grandes centros regionales.

\section{EL DESARROLLO DE LOS ESPACIOS RURALES Y DE BAJA DENSIDAD}

El envejecimiento de la población y la existencia de extensos territorios de baja densidad, con dinámicas negativas hace necesaria la puesta en marcha, con mayor energía y recursos que hasta ahora, de medidas de apoyo a la conciliación de tiempo laboral y familiar y de incentivos a la natalidad, para enfrentar un problema común grave en el Noroeste de España y no solo en sus áreas rurales. Galicia, Asturias y Castilla y León presentan una continuidad de la situa- 
ción de desequilibrio entre defunciones y nacimientos, mientras que País Vasco, Cantabria, Navarra y La Rioja se encuentran en un estado próximo al estancamiento demográfico; apoyándose el crecimiento actual, en todos los casos, en la inmigración.

La existencia de las mencionadas rupturas territoriales, que configuran territorios en declive y ruralidad en transformación, dibuja un panorama con profundas disparidades territoriales. El impacto de la inmigración y la tendencia a la mejora de la natalidad abren perspectivas menos negativas para el futuro a medio plazo, que deben ser reforzadas desde el ámbito de las políticas de conciliación, arbitradas en el marco de acuerdos entre la administración, los agentes sociales y las empresas y desde la mejora de la cobertura de los servicios públicos, en particular en el campo de la infancia y de la población anciana, cuya atención se presenta además como un importante nicho de empleo joven.

Otro aspecto que se ha revelado fundamental, no tanto en la cantidad de recursos utilizados como en la apertura de nuevas vías, son las políticas y programas de desarrollo rural de alcance europeo, cuya continuidad debe apoyar el sostenimiento de las tendencias a la diversificación de actividades, el proceso de terciarización económica, el aumento de las relaciones entre la ciudad y los territorios rurales y la difusión de flujos crecientes desde las áreas metropolitanas.

La ordenación de estos territorios se puede afrontar desde varias ópticas: la de la accesibilidad, el reequilibrio territorial, la interrelación con el sistema urbano, la diversificación productiva, la potenciación de los pequeños núcleos urbanos de área de influencia comarcal, la mejora de los servicios y equipamientos públicos, que conllevan una mayor dotación de recursos y la revitalización demográfica y social.

La necesidad de refuerzo de los pequeños centros urbanos y de los núcleos rurales se apoya en la existencia de algunas significativas tendencias demográficas positivas y de incremento de la oferta de servicios y de la cualidad residencial de los centros comarcales, como último escalón del sistema urbano; los cuales evidencian una mejora del nivel de vida de sus residentes, asociada al nuevo empleo y a la revitalización del pequeño tejido empresarial, a la mejora de la accesibilidad y al incremento de la relación con los centros urbanos del siguiente nivel jerárquico y a la mejora de equipamientos y servicios públicos. Son núcleos urbanos de pequeño tamaño (2.000-15.000 habitantes) en los que cualquier iniciativa tiene un gran impacto potencial y adquiere gran dimensión en sus efectos inducidos. 
Figura 3. Densidad de población en las regiones del espacio atlántico europeo
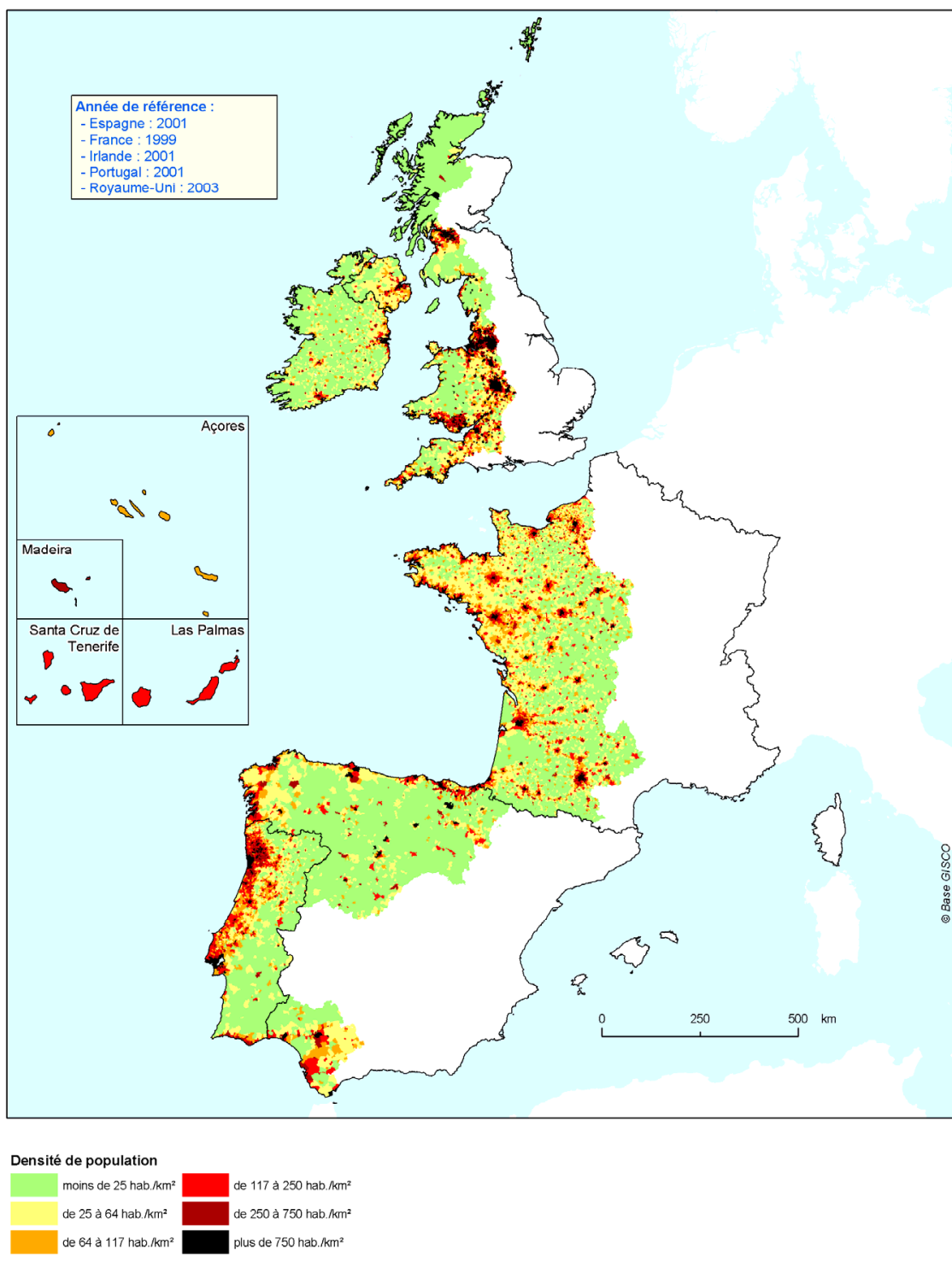

Las tendencias más positivas se han producido en las comunidades autónomas más pequeñas, en las que la atención a este último escalón urbano ha sido mayor en cuanto a mejora de la accesibilidad, integración en el sistema territorial, mejora de los servicios públicos, infraestructuras y equipamientos, ordenación 
del territorio, aprovechamiento de los efectos inducidos por las declaración de espacios protegidos, promoción de suelo empresarial y desarrollo de programas e iniciativas de desarrollo. La continuidad de estas políticas significa el refuerzo de estos núcleos, vitales para la supervivencia del mundo rural y para evitar el despoblamiento de importantes áreas interiores y de montaña.

La promoción de nuevas actividades y las respuestas a los cambios de la agricultura, así como los programas de desarrollo rural puestos en marcha durante las dos últimas décadas, han dado lugar a importantes cambios en la agricultura (especialización, descenso de empresas, incremento de la productividad, pérdida de empleo) y han creado nuevos escenarios de diversificación, favoreciendo el turismo rural y de naturaleza (vinculado o no a espacios protegidos), a políticas de ordenación del territorio y a nuevos equipamientos vinculados a los espacios protegidos, a las nuevas actividades de transformación agrarias, etc. Se hace necesaria una continuidad en el apoyo a los procesos de desarrollo rural y de protección de la actividad en las áreas rurales más frágiles (montaña, espacios alejados o de baja densidad) mediante líneas específicas de financiación. La resolución de los problemas de aislamiento implica nuevas oportunidades de relación entre el mundo urbano y rural, a través del papel intermedio de los centros comarcales y pequeñas ciudades y de la captación de crecientes flujos urbanos sobre los que sustentar nuevas actividades, vinculadas o no a los recursos del territorio y a la valorización de las producciones locales de calidad.

\section{LA MEJORA DE LA CONECTIVIDAD INTERNA Y EXTERNA}

La posición periférica de buena parte del territorio atlántico ha inducido históricamente condiciones de baja accesibilidad, reforzadas por la dependencia de los sistemas de infraestructuras respecto a los principales nodos peninsulares. Esta situación ha mejorado sustancialmente entre 1985 y 2010, con la construcción de los grandes ejes radiales de autovías, las primeras líneas de ferrocarriles de alta velocidad, el incremento de tráfico de los aeropuertos regionales y la mejora de los puertos marítimos. Sin embargo, solo en los últimos años se ha abordado la mejora de las comunicaciones transversales que enlazan directamente los principales nodos urbanos atlánticos.

La puesta en marcha por el gobierno español del Plan Estratégico de Infraestructuras y Transporte (PEIT), con su marco financiero correspondiente y la inclusión de los principales corredores de transporte en la red europea, parecen despejar dudas, a pesar de los cambios en la obtención de financiación comunitaria a partir de 2008, sobre la continuidad de los proyectos en infraestructuras de transporte y de la construcción de las líneas ferroviarias de alta velocidad, fundamentales para la integración del espacio atlántico. Las medidas de mejora 
de la conectividad internacional de las regiones atlánticas españolas se enmarcan así en el desarrollo del PEIT entre 2005 y 2020.

Desde el punto de vista de las conexiones internacionales, los transportes aéreos y marítimos adquieren una importancia fundamental. Las mejoras previstas miran a un refuerzo del sistema de puertos y a la realización de grandes inversiones de mejora y ampliación en función de sus especializaciones actuales, así como el impulso de nuevas rutas complementarias y la consolidación de las autopistas del mar. Así, se desarrollan importantes proyectos de ampliación, mejora y/o construcción de puertos externos en Ferrol, A Coruña, Gijón y Bilbao, entre otros. Sin embargo, parece que no hay una correspondencia nítida entre la potencia instalada y los tráficos actuales, por lo que debería realizarse un análisis pormenorizado de los potenciales de cada puerto y sus posibilidades de crecimiento, superando las especializaciones actuales y la mera continuidad de las mismas en el escenario previsto en el PEIT. Existe un número relevante de puertos bien equipados, vinculados a áreas metropolitanas y a sistemas intermedios e incluso a ciudades medias. Infraestructuras que presentan importantes potenciales de crecimiento y que pueden servir de base a un incremento de la participación del trasporte marítimo en el transporte general de mercancías en la fachada atlántica europea. La potenciación de la relación entre los puertos se presenta como una necesidad estratégica para el desarrollo del transporte marítimo y para evitar la continuidad de la tendencia actual que conduce a la saturación del sistema de transporte por carretera. Castilla y León no es ajena a la actividad de los grandes puertos atlánticos que tienen aquí infraestructuras básicas y logísticas imprescindibles para asegurar la intermodalidad del transporte en los grandes ejes de relación de Europa.

Hay que constatar la importancia de incardinar el sistema de puertos atlánticos españoles en la red europea de autopistas del mar, que, en el marco del Libro Blanco del Transporte, persigue la potenciación de la navegación de cabotaje en detrimento del transporte por carretera. En este sentido, la alternativa de los puertos atlánticos de España, para evitar la congestión del paso de IrúnHendaya y sur de Francia, puede considerarse como una opción muy razonable para la exportación (industria automotriz, de bienes de equipo, maquinaria, productos agroalimentarios...) hacia el resto de Europa. Situación similar a la relación con los puertos de Portugal y a la actual situación del transporte de mercancías entre España y Portugal, dominada también por el transporte por carretera.

Respecto al transporte aéreo, el sistema de aeropuertos regionales españoles se ha caracterizado en la última década por un incremento de la actividad, número de vuelos, conexiones internacionales y volumen de pasajeros. Sin embargo, el transporte aéreo en España sigue presentando el rasgo de la excesiva 
dependencia respecto a los aeropuertos de Madrid y Barcelona, en un nivel próximo a la saturación, a pesar de las repetidas ampliaciones. El PEIT no hace sino refrendar esta situación apostando por una continuidad estructural del sistema de aeropuertos regionales. Sin embargo, el fuerte impacto de las líneas de bajo coste y la ampliación de la oferta de vuelos internacionales y regionales, junto con la respuesta de las aerolíneas tradicionales, con el incremento de la oferta de líneas directas entre sistemas urbanos intermedios y de líneas internacionales desde aeropuertos regionales, está transformando rápidamente el panorama del transporte aéreo, de modo que el PEIT probablemente esté apostando por una opción estratégica ya superada por la realidad, a pesar del actual escenario de crisis.

La saturación de Madrid y Barcelona se une a las grandes posibilidades de crecimiento de los principales aeropuertos regionales y de las relaciones directas entre ellos. Las medidas a tomar deberían orientarse a una mejora de equipamientos en los aeropuertos regionales, en la continuidad del incremento de las relaciones internacionales con una diversificación de destinos tradicionales (Londres, París, Bruselas, Frankfurt), sobre la base de la apertura de nuevas relaciones y nuevas conexiones vinculadas. El transporte aéreo interregional tiene como perspectiva a medio plazo, en España, la competencia del tren de alta velocidad, por lo que probablemente deberá reorientarse a conexiones peninsulares extremas (distancia en torno o superior a $1.000 \mathrm{Km}$, islas, turísticas) y, sobre todo, a aumentar las conexiones internacionales, no solo con las principales ciudades europeas y las interconexiones que éstas ofrecen, sino a un abanico más amplio de ciudades, particularmente las atlánticas, como resultado del incremento de relaciones directas entre ellas.

El ambicioso plan ferroviario de alta velocidad, con una red básica en funcionamiento (Madrid-Sevilla y Málaga, Madrid-Valladolid, Madrid-ZaragozaBarcelona), necesita articularse internacionalmente. Desde esta óptica se debe avanzar con agilidad en la conexión Valladolid-Burgos-País Vasco-Francia, así como en su articulación con el valle del Ebro. La construcción del AVE Madrid-Zamora-Vigo-A Coruña debe impulsar la vinculación del eje costero gallego con el eje atlántico Lisboa-Porto. La conexión Madrid-Valladolid (Medina del Campo)-Salamanca necesita su continuación hacia Portugal, al tiempo que se completa la vinculación hispano-portuguesa a través de Sevilla-HuelvaAlgarve y de Madrid-Lisboa. La posición intermedia de Castilla y León va a favorecer que la mayor parte del sistema urbano principal quede comunicado por la red ferroviaria de alta velocidad en los próximos años, con un horizonte más lejano para Ávila y Soria.

Las líneas en construcción tienen una importancia fundamental para la mejora de tiempos de viaje y condiciones del mismo, no solo en las conexiones direc- 
tas, sino también por su papel intermedio en otra rutas entre Madrid y la periferia peninsular, en las que está prevista la construcción de nuevas líneas de alta velocidad, bien exclusivas de viajeros (Madrid-Galicia) o mixtas (el resto de las conexiones indicadas). Así, la línea de Valladolid está en obras en la variante de Pajares y en la provincia de León, con un horizonte de finalización en 2013. Está en construcción la línea entre Valladolid y Palencia hacia Cantabria y País Vasco. La línea Madrid-Zamora-Galicia está en obras en casi todos los tramos; más atrasada va la de Medina del Campo-Salamanca, estando en estudio su continuidad hacia Portugal. En cualquier caso, y aunque la culminación de estos proyectos se dilate, en las previsiones del PEIT, hasta 2015-2020, la apertura de tramos clave, como Madrid-Valladolid posibilita una importante reducción de tiempos de viaje entre Madrid y el conjunto de las ciudades castellano-leonesas y atlánticas, mejorando la función, muy debilitada hasta ahora, del tren en el transporte de viajeros.

En transporte de mercancías, el tren ha continuado perdiendo en favor de la carretera. La recuperación de su papel se apoya, en el PEIT, en la utilización mixta de las líneas de altas prestaciones previstas y en el uso exclusivo de líneas que queden fuera del circuito de pasajeros o que ya están en desuso en la actualidad y cuya reutilización está en estudio, caso de la línea Astorga-ZamoraSalamanca (Ruta de la Plata). En cualquier caso la tendencia a la saturación de las principales vías de transporte por carretera hace necesaria una revisión del papel del ferrocarril y de su potencialidad, como alternativa de futuro, reutilizando vías en desuso, las líneas convencionales alternativas al AVE o las escasamente utilizadas.

La conexión internacional de la red de autopistas y autovías se realiza a través de diversos enlaces. Desde el punto de vista transnacional destaca la saturación del paso de Irún-Hendaya, al que habría que responder con nuevas alternativas. La relación con Portugal ha mejorado con la práctica culminación de la autovía entre Salamanca-Ciudad Rodrigo-Aveiro, e impulsa nuevas relaciones como Verín-Chaves y Zamora-Bragança. La red de autopistas y autovías presenta una situación casi concluida en el sistema radial de relación con Madrid y bastante más incompleta en las relaciones transversales entre los distintos sistemas urbanos. La necesidad de ejes transversales demanda la finalización de la Ruta de la Plata; del eje de Castilla en la frontera portuguesa; del Benavente-PalenciaBurgos; del eje del Duero (Valladolid-Aranda de Duero-Soria-Tudela), y de la relación directa entre León y Valladolid.

Gran importancia estratégica tiene la Ruta de la Plata. Este eje estructuraría el gran vacío territorial entre el interior de Galicia y Asturias y el norte de Huelva, suturando dicho espacio en sentido N-S y en el espacio fronterizo con Portugal. Trenzaría una larga serie de áreas metropolitanas, sistemas urbanos, villas y pe- 
queñas ciudades que en la actualidad viven relativamente aisladas o con escasa relación entre sí, con el añadido de la presencia de la frontera: desde Asturias al espacio bético, pasando por León, Astorga, Benavente, Zamora, Salamanca, Guijuelo y Béjar hacia Sevilla y Huelva.

La mejora de la integración interna se basa en el aprovechamiento de la rápida creación de infraestructuras de transporte en curso. Y ello a través de dos líneas fundamentales de actuación: la mejora de la conectividad interna de las áreas metropolitanas, sistemas urbanos intermedios y red urbana en general, y la vertebración de los grandes ejes de relación entre los nodos de la red urbana y la comunicación de las áreas más aisladas o con peores condiciones de accesibilidad. La congestión de la áreas metropolitanas exige medidas de accesibilidad interna que pasan por la mejora de los ferrocarriles metropolitanos y transportes urbanos en general, los servicios ferroviarios de cercanías, la potenciación de los aeropuertos y la mejora de la malla de autovías y grandes vías urbanas, poniendo en valor vías alternativas en distancias medias. En general, el proceso de crecimiento de las áreas metropolitanas y sistemas urbanos intermedios, junto a la densificación de las regiones urbanas, demandan en los próximos años un singular esfuerzo de optimización y construcción de nuevas infraestructuras metropolitanas e interurbanas, en particular con actuaciones en el capítulo del transporte público (tranvías, ferrocarril metropolitano y de cercanías, autobús) que aporten soluciones alternativas al incremento continuado del tráfico urbano y al uso masivo del vehículo privado.

Por otro lado, la generalización del uso de las tecnologías de la información y de la comunicación exige aún esfuerzos para facilitar el acceso masivo de la población y de las empresas. Para ello es necesario el refuerzo de las medidas que alienten la competencia entre empresas suministradoras así como el incremento de la formación, el conocimiento y la utilización de las distintas tecnologías. Es importante la extensión de servicios y medios al conjunto de la población, con la inclusión de las pequeñas ciudades y de las áreas rurales en condiciones de igualdad con las ciudades principales. La dotación de centros educativos, centros sociales, organismos de la administración y empresas se revela como primordial para su desarrollo y para la igualdad de oportunidades de las poblaciones y territorios.

El acceso de la población a las TIC y la conexión a las redes informáticas se ha generalizado a lo largo de las dos últimas décadas por lo que las medidas conciernen más al campo de difusión y utilización de las TIC que a la extensión de cobertura y redes. En este capítulo los próximos retos son la extensión de las nuevas tecnologías en materia de telefonía móvil, la generalización de las líneas telefónicas y de Internet de alta velocidad y el incremento del uso de las redes para actividades económicas y de intercambio, así como en la relación de la po- 
blación con la administración. La definitiva implantación de la televisión digital e interactiva y la diversificación de fuentes y servicios avanzados a empresas y particulares abren campos de actividad en continua transformación y expansión $\mathrm{y}$ vinculados a las actividades de $\mathrm{I}+\mathrm{D}+\mathrm{I}$.

\section{LAS POLÍTICAS DE APOYO A LA INNOVACIÓN, INVESTIGA- CIÓN Y DESARROLLO}

El desarrollo y la extensión de las nuevas tecnologías de la información y la comunicación ha seguido una pauta ascendente y muy rápida en los últimos años que necesita ser reforzada en algunos aspectos; en particular en lo que concierne a su utilización para el refuerzo de las redes y nodos de investigación, desarrollo e innovación y de la relación entre empresas, centros de investigación y universidades, con apoyo expreso a los polos de intercambio existentes y apertura de nuevas vías, a través de planes y convocatorias específicos. Las líneas estratégicas de futuro apuntan a la creación y refuerzo del sistema de polos y parques tecnológicos, vinculados o no a centros universitarios, y a las plataformas de intercambio entre centros de investigación y sistema productivo. También hacia el impulso del uso de las TIC en las pequeñas y medianas empresas, como herramienta para sus actividades de producción, comercialización e intercambio y vinculación de éstas en redes de cooperación empresarial y de fomento de la investigación. El aprovechamiento integral de la redes de organismos de cooperación entre universidades y empresas, oficinas de transferencias de resultados de investigación debe ser paralelo al impulso de la formación de investigadores, de los contratos tecnológicos y de la evaluación de los centros universitarios y de investigación, así como de la constitución de grupos de excelencia en investigación. Es necesario, por último, el refuerzo de la presencia de los centros de investigación en redes trasnacionales de I+D+I y en proyectos internacionales.

La existencia de experiencias y la evaluación de políticas y resultados de las mismas en torno a la creación de polos de investigación, desarrollo, innovación e intercambio permite definir políticas de refuerzo de su papel motor en las economías regionales. El ejemplo de los polos o parques tecnológicos existentes en Valladolid, Bilbao, Asturias o Galicia debe ser aprovechado para un más intensivo seguimiento de sus actividades y aportación de recursos, a través de políticas específicas de fomento de la investigación. La existencia de una estructura empresarial de cierta dimensión en cada uno de los polos mencionados establece la masa crítica necesaria para el fomento de las relaciones de intercambio entre las empresas y los centros de investigación y para el reforzamiento de los polos de $\mathrm{I}+\mathrm{D}+\mathrm{I}$ existentes y futuros. Es una necesidad patente, una vez avanzado el proceso de creación de infraestructuras para el desarrollo, centrar esfuer- 
zos en lo que hoy aún es una insuficiencia en la España atlántica y que por ello debe tener una prioridad fundamental, como punto más débil de la actual estrategia de desarrollo.

La presencia de centros universitarios y de investigación de relevancia, una estructura empresarial densa, empresas de ámbito mundial y una población relativamente numerosa con formación avanzada, avala la política de apoyo a la creación de polos de excelencia en I+D+I en el País Vasco, Asturias, Galicia, Valladolid, Burgos, León o Salamanca, a partir de las experiencias existentes de parques tecnológicos, polos universitarios y escenarios de intercambio y cooperación empresarial.

Los sistemas productivos atlánticos se han caracterizado hasta época reciente por una especialización en actividades industriales tradicionales, industrias metalúrgicas, químicas, extractivas, energéticas, con un proceso de modernización y reestructuración dilatado. Este proceso ha dado lugar a problemas graves para el empleo y las dinámicas locales, así como para el crecimiento de estas regiones, pero también constituyó una oportunidad por los importantes procesos de modernización llevados a cabo en sectores como la siderurgia, la metalurgia, la industria química, la automoción o la agroalimentaria, a continuar y reforzar en los próximos años para consolidar el proceso. Sin embargo, la difusión de las actividades de las grandes empresas y la constitución de sistemas locales y distritos industriales ha sido débil, salvo en el caso del País Vasco y sus regiones vecinas, así como la formación de redes de cooperación empresarial.

Por ello, las políticas a desarrollar en los próximos años pasan por la habilitación de puntos de encuentro y cooperación, apoyo a la modernización y cooperación de los sistemas de pequeñas y medianas empresas, vinculación entre las grandes empresas multinacionales industriales y de servicios y el sistema de pequeñas y medianas empresas de ámbito local. El proceso de tecnologización e internacionalización de los sistemas locales de pequeñas y medianas empresas se revela como fundamental, si tenemos en cuenta además su papel en el crecimiento del empleo. La tendencia a la pérdida de la ventaja comparativa en precios y costes laborales respecto a otros países europeos necesita de un refuerzo de las políticas de aumento de la competitividad de las empresas y de los recursos empleados en investigación, desarrollo e innovación como vía de integración plena en el mercado mundial en condiciones de competitividad.

La generalización del acceso a la enseñanza superior, en porcentajes elevados entre la población menor de 40 años, y el fundamental papel que la disponibilidad de un numeroso grupo de población con formación superior ha significado en el crecimiento español de las dos últimas décadas, deja paso a una nueva situación marcada por la reforma de las enseñanzas superiores en Europa, que se- 
ñala una oportunidad para la modernización y armonización del sistema de enseñanza e investigación universitario (proceso de Bolonia, espacio europeo de educación superior)

Esta oportunidad debe dar paso a una mejora generalizada del sistema de educación superior, superada la época de masificación y extendida la oferta de estudios sobre el territorio hasta el nivel de las ciudades medias-pequeñas, con la mejora de los centros, su vinculación a centros tecnológicos avanzados y el incremento de la relación con las empresas y el sistema productivo en general. La introducción de nuevos escenarios de competencia entre universidades, de aplicación de sistemas rigurosos de evaluación de la actividad y la producción científica, así como la mejora en los sistemas de selección y formación de profesores e investigadores se plantean como retos para la universidad española, ante los que se enfrenta con situaciones de partida diferenciadas entre los distintos centros, más o menos anclados en prácticas tradicionales o abiertos a las nuevas funciones de cooperación con la sociedad y el territorio en el que se localizan.

Otro campo de trabajo es el de la inserción laboral de los titulados, en particular de la mujeres, que se ha visto dificultada en las regiones menos dinámicas y en los centros menos innovadores, abriendo procesos de captación de titulados por parte de las regiones más dinámicas, con la pérdida de capital social correspondiente. La situación actual de los mercados de trabajo en las regiones atlánticas, demográficamente estancadas, plantea importantes problemas de mano de obra, de mayor o menor cualificación, en muchas actividades (agricultura intensiva, construcción, hostelería, servicios a la población...), lo que ha redundado en un incremento muy notable de la inmigración. Se debe afrontar la situación desde el sistema de formación profesional reformado en 1990, con captación de un porcentaje mayor de población estudiantil y mediante la continuidad de los flujos inmigratorios que, en las regiones atlánticas, van a seguir siendo necesarios (entre el 2 y el $8 \%$ de la población total, según regiones), si tenemos en cuenta los ritmos demográficos y su lenta recuperación, para poder mantener los actuales niveles de actividad y empleo.

Esto plantea importantes interrogantes sobre el futuro del sistema de captación de mano de obra extracomunitaria y su adecuación legal. En cualquier caso, esta situación va a requerir del mantenimiento de la estructura de formación profesional ocupacional, reorientado a la población inmigrante. Y ello para el correcto aprovechamiento de la formación de la población, del capital social y de la capacidad del sistema productivo. 


\section{LA ARTICULACIÓN TRANSNACIONAL ENTRE ESPACIOS ATLÁNTICOS DE DESARROLLO}

Desde el punto de vista atlántico español, el refuerzo de las relaciones entre territorios transfronterizos se afronta en varios espacios de cooperación: Norte de España-Suroeste de Francia, eje costero Galicia-Portugal, Algarve-Andalucía Atlántica y el área fronteriza interior de Portugal y Castilla y León

Las recomendaciones para el reforzamiento de la vinculación transfronteriza apuntan a una mejora de la permeabilidad fronteriza que posibilite el aprovechamiento de las oportunidades que ofrece un mallazo de relaciones más denso entre los nodos urbanos de distinto nivel en ambos lados de la frontera y limite sus efectos negativos. Los nodos fundamentales de este espacio están constituidos por el sistema de ciudades intermedias, para las que la apuntada permeabilización fronteriza demanda una mejor comunicación y conexión. Un hito inmediato de futuro lo constituye la conexión mediante líneas de alta velocidad entre Valladolid y Burdeos.

Gran importancia adquiere como espacio motor el Suroeste de Francia-Norte de España. Este presenta gran actividad, mejores indicadores relativos de desarrollo y mayor densidad urbana, y se extiende por Cantabria, País Vasco, La Rioja, Navarra y el eje de Castilla, sobre los nodos de Miranda, Burgos (eje Paris-Madrid) y de Valladolid-Palencia. Se aprecia, también, la recuperación del tradicional eje industrial, hoy en fase de revitalización, del norte de España (País Vasco-Cantabria-Asturias), con un crecimiento extendido a los espacios litorales interurbanos. Sus indicadores son positivos y tienden a la articulación creciente del mencionado eje cantábrico y del eje transnacional entre Francia, España y Portugal, constituyendo corredores dinámicos que han impulsado el crecimiento de sus nodos urbanos principales.

Los procesos de difusión se extienden a otros ejes del subespacio motor franco-español, situados en áreas de menor densidad e indicadores de desarrollo más limitados: Valladolid-León-Asturias, Valladolid-Benavente-Galicia, definidos como espacios de integración de fuerte potencial crecimiento. La orla montañosa de Castilla y León implica áreas de baja densidad que dificultan la conexión efectiva de los espacios definidos entre las regiones costeras e incluso con Madrid, introduciendo dificultades a la implantación de la red de infraestructuras de transporte. Del mismo modo, la ruptura territorial mencionada, en el oeste de Castilla y León, supone un reto para la articulación de este espacio con el correspondiente al eje atlántico de Galicia y Portugal. Trascendental importancia tiene la conexión con uno de los ejes de mayor crecimiento en las dos últimas décadas: el del Ebro, que para este espacio motor tiene una importancia tan decisiva como Madrid. 
Figura 4. Índice de desarrollo económico relativo (SDEA 2005)

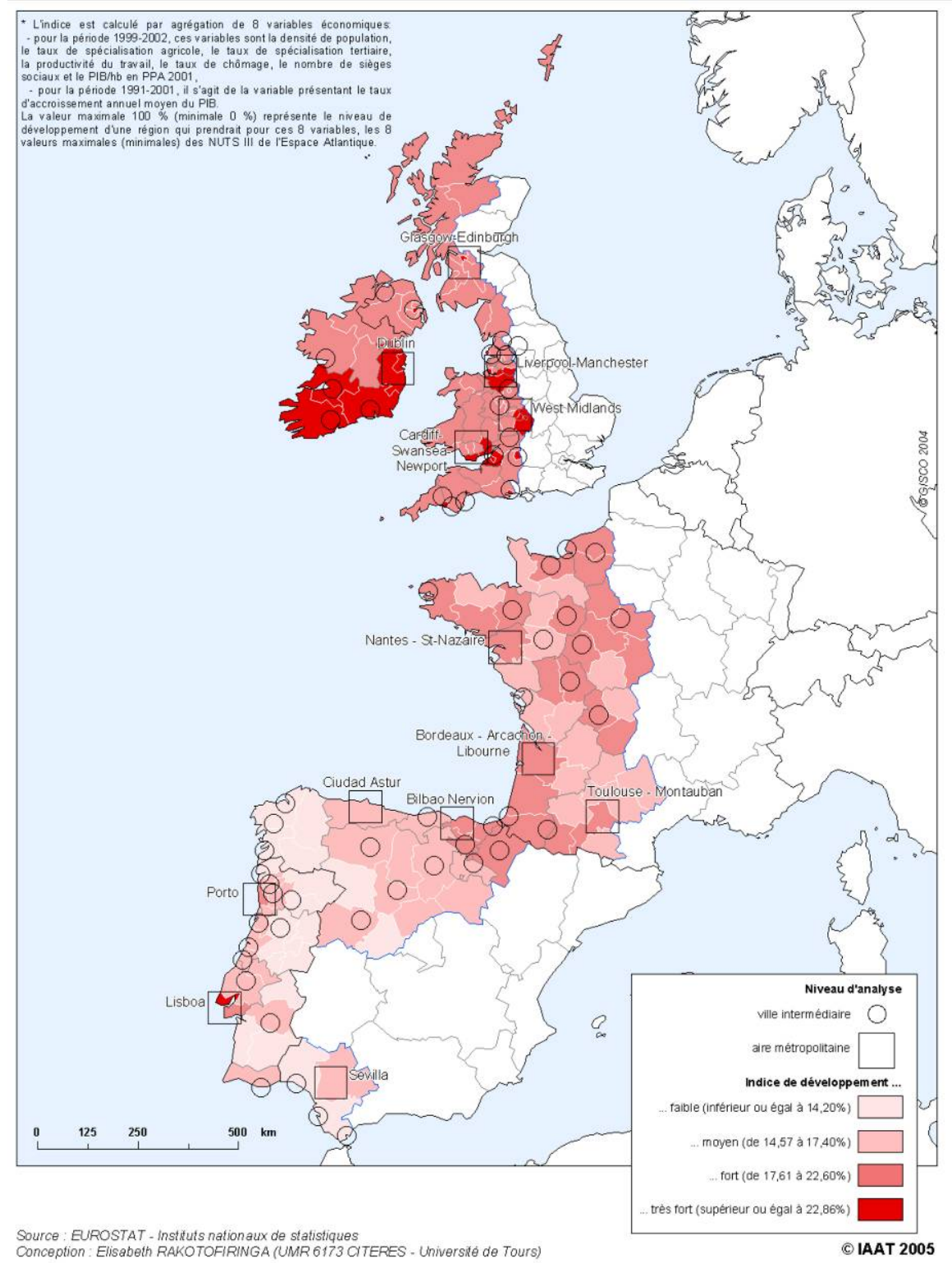

Respecto al espacio de integración de Castilla y León se manifiesta una mayor vitalidad en torno al eje Vitoria-Burgos-Palencia-Valladolid; presentando de nuevo, como en el caso del Cantábrico, un gradiente negativo hacia el oeste, en la conexión con Salamanca y Portugal (Guarda-Aveiro y el eje Porto- 
Lisboa). Este espacio debería prolongarse hacia su integración con Portugal por el oeste, hacia el norte, con León y hacia el sur, con la presencia dominante de Madrid. Sus fortalezas y debilidades son distintas a las del espacio cantábrico, no se ha producido aquí una reconversión larga y hay una diversificación productiva notable. Se debe aprovechar su potencial de crecimiento, disponibilidad de suelo, existencia de grandes ejes de infraestructuras y posición intermedia entre nodos urbanos de primer y segundo nivel. Especial atención requiere la puesta en valor de su industria cultural y turística, vinculada a universidades de prestigio y al riquísimo patrimonio cultural del que son exponentes principales el camino de Santiago (extendido por la mayoría de las regiones atlánticas, desde Navarra a Galicia) y las ciudades y monumentos patrimonio de la humanidad (Salamanca, Ávila, Segovia, Santiago...)

Las consecuencias de la construcción del policentrismo se orientan a la reafirmación de una red tupida de ciudades y polos que facilite la puesta en valor y la relación entre todos los nodos del territorio y provoque la emergencia de algunos polos que, especialmente en Castilla y León, pueden contribuir al equilibrio territorial: es el caso ya mencionado del espacio motor regional de Valladolid- Palencia-Tordesillas, como gran área urbana castellana (confluencia de autopistas, aeropuerto emergente y redes de alta velocidad, así como una cierta dimensión demográfica), pero también de algunas ciudades de localización estratégica y atracción de empresas industriales o de servicios y en particular logísticas, como Burgos, Benavente, Miranda de Ebro, Aranda de Duero, Ponferrada, Salamanca o León, que pueden ver potenciada su condición nodal, en sus diferentes niveles. La ordenación del oeste de Castilla y León necesita actuaciones para sustentar nuevos procesos de generación de actividad y desarrollo. En este sentido convendría repensar la estructura y alcance del sistema de ciudades castellanoleonesas y su papel como polos impulsores del desarrollo y de la ordenación del territorio, más desde una óptica interregional y europea que interna. Por último, hay que reseñar la importancia de las pequeñas ciudades que ejercen el papel de último escalón del sistema urbano como centros comarcales. Importancia que se podría englobar en el concepto de "microcentrismo", la capacidad estructurante sobre el territorio "rural" de muchas villas y pequeñas ciudades que muestran indicadores demográficos y económicos positivos.

Las posibilidades de cooperación en el impulso a la integración de la fachada atlántica ibérica y Castilla y León son muy amplias y deben ser exploradas desde un objetivo fundamental, buscar escenarios que permitan un crecimiento sostenido de la actividad a partir de la articulación territorial de un espacio periférico con problemas de accesibilidad debido a su localización excéntrica, desfavorable respecto a los principales ejes de crecimiento de la península Ibérica. 


\section{BIBLIOGRAFÍA}

BLANCO FERNÁNDEZ, J. (2004): La emergencia de las nuevas ciudades en la era global. Gijón, Trea.

CABERo DiegueZ, V. (1982): El espacio geográfico castellano-leonés. Valladolid, Ámbito.

Cabero Dieguez, V., Troitiño Vinuesa, M. A., Calavía Redondo, M. y Llorente PINTO, J. M. (1990): Las comarcas tradicionales, en A. CABO y F. MANERO (dirs.) Geografía de Castilla y León. Valladolid, Ámbito, tomo 8.

CAmpesino, A. J.; Troitiño, M. Á. y CAMPos, M. L. (coords.) (1995): Las ciudades españolas a finales del siglo XX. Murcia, Grupo de Geografía Urbana de la Asociación de Geógrafos Españoles-Universidad de Castilla-La Mancha.

Delgado Viñas, C. (ed.) (2010): La Montaña cantábrica oriental. Santander, Estudio.

JUARISTI LINACERO, J. (1995): «El sistema urbano español y sus relaciones con el sistema urbano europeo», en A. J. CAMPESINO, M. A. TROITIÑo y M. L. CAMPOS (coords.) (1995): Las ciudades españolas a finales del siglo XX, Murcia, Grupo de Geografía Urbana de la A.G.E. Universidad de Castilla-La Mancha, 17-27.

Junta De CASTILla y LeÓn (2001): Plan de desarrollo Regional de Castilla y León. 2000-2006. Consejería de Economía y Hacienda.

JunTA DE CASTILlA Y LEÓN (1995): Atlas del territorio de Castilla y León. Consejería de Medio Ambiente y Ordenación del Territorio.

JunTA De CASTILla y LeÓn (2000): Directrices de Ordenación del Territorio de Castilla y León. Consejería de Medio Ambiente y Ordenación del Territorio.

LOPEZ Trigal, L. (1987): Geografía humana de Castilla y León. Barcelona, Oikos-tau.

LÓPEZ TRIGAL, L. (1995): «El sistema urbano de Castilla y León en relación con la prospectiva de la fachada atlántica», Medio Ambiente en Castilla y León, n 3, 1620.

MENÉNDEZ FERNÁNDEZ, R. (2009): «La red urbana de Castilla y León», en F. RODRÍGUEZ GUTIÉRREZ (ed.), Áreas metropolitanas de España. La nueva forma de la ciudad. Oviedo, Universidad de Oviedo.

MiNISTERIO DE FOMENTO (2005): Plan Estratégico de Infraestructuras y transporte 2005-2020. Madrid

RODRÍGUEZ GutiÉRREZ, F. y MENÉNDEZ FERnÁNDEZ, R. (2005): Geografía de Asturias. El proceso de reestructuración territorial de una región de tradición industrial. Barcelona, Ariel.

RODRÍGUEZ GUTIÉRREZ, F. y otros (2006): Coastatlantic. Integrated Coastal Zone Management: Towards an Atlantic Vision. Oviedo, Universidad de Oviedo - Gobierno del Principado de Asturias - Conseil General de la Gironde.

SANTOS Y GANGeS, L y PEIRET I CARRERA, A. (2001): «Articulación regional y comarcas en Castilla y León: las directrices de ordenación del territorio». Boletín de la Asociación de Geógrafos Españoles, ${ }^{\circ} 32,177-190$.

VARIOS AUTORES (2002): Study on the Construction of a Polycentric and Balanced Development Model for the European Territory. Étude sur la Construction d'un Modèle de Développement Polycentrique et Équilibré pour le Territoire Européen. Santa María da Feira, Conference of Peripheral Maritime Regions of Europe. 
VARIOS Autores (2005): Atlantic Spatial Development Perspective. Schema de developpement de l'espace atlantique. Santa María da Feira, Conference of Peripheral Maritime Regions of Europe.

Velarde, J., GARCia Delgado, J. L. y Pedreño, A. (1992): Ejes territoriales de desarrollo. España en la Europa de los noventa. Madrid, Economistas Libros. 